molecular structure of one of these tailored proteins while it was bound to the flu haemagglutinin, revealing atomic-level accuracy in the designed interaction.

Science 332, 816-821 (2011)

\section{IMMUNOLOGY}

\section{Blocking brain} inflammation

Harmful brain inflammation triggered by a subset of immune cells can be quelled by the action of a hormone on an oestrogen receptor.

Microglia are immune cells that trigger inflammation in the central nervous system and carry an oestrogen receptor called ER $\beta$. Christopher Glass and Kaoru Saijo at the University of California, San Diego, and their team screened a panel of molecules that bind to $\mathrm{ER} \beta$ for their ability to block inflammation in microglia. They found that a few synthetic chemicals, as well as a natural steroid hormone called ADIOL, activate ER $\beta$, kicking off a cascade of reactions that ultimately prevents inflammation. ADIOL also protects mice from an autoimmune condition similar to multiple sclerosis.

The authors suggest that drugs that stimulate this pathway could be used to treat neurodegenerative and autoimmune diseases.

Cell 145, 584-595 (2011)

\section{SEISMOLOGY}

\section{Awakening a fault line within}

A large fault section off Sumatra that had been seismically dormant for more than 30 years has recently reawakened, thanks to a series of large earthquakes in the area during the past decade.

Kelly Wiseman at the University of California, Berkeley, and her team linked data derived from the Global Positioning System on surface motions from Sumatran stations with the known geometry and mechanisms of recent quakes. They found that a 900-kilometre-long 'backthrust' - arising from the longer Sunda megathrust fault that caused the 2004 Indian Ocean earthquake and tsunami - produced a moderate quake in 2005 and another in 2009.

If a rare faulting event were to rupture the newly active thrust, it could produce a quake on the order of magnitude 8.5, and, potentially, a large tsunami, the authors suggest. Geophys. Res. Lett. doi:10.1029/ 2011GL047226 (2011)

\section{PHYSICS \\ Achieving spin control}

By combining an optical microscope with an atomic force microscope (AFM), researchers have imaged individual electronic spins with high resolution.

Amir Yacoby and his team at Harvard University in Cambridge, Massachusetts, implanted clusters of nitrogen ions into a diamond sample, creating individual spins. They applied a magnetic field gradient to the spins by passing the magnetized tip of the AFM over the sample. This allowed them to draw a map of the sample's individual spins in three dimensions. The authors showed that it would be possible to resolve spins just 9 nanometres apart.

The system could be used for studies of fundamental physics, because the set-up allows quantum control and manipulation of individual spins.

Nature Phys. doi: 10.1038/

nphys1999 (2011)

\section{AUTOIMMUNITV}

\section{Taming psoriasis with vitamin D}

Vitamin D may ameliorate the symptoms of the inflammatory skin disease psoriasis by enhancing the production of a molecule that blocks the assembly of inflammatory complexes in the skin.

COMMUNITY CHOICE

The most viewed papers in science

\title{
A search for depression genes
}

\section{Highly Read \\ on cell.com \\ 10 April-10 May}

A genome-wide analysis of more than 15,000 people has revealed an association between a gene and major depression.

Martin Kohli and Elisabeth Binder at the Max Planck Institute of Psychiatry in Munich, Germany, and their colleagues first compared the genomes of 353 patients with depression with those of 366 controls. They teased out a gene, SLC6A15, that was strongly associated with depression, and went on to replicate this finding in six other independent groups of patients. The gene encodes a transporter protein that moves certain amino acids across the cell membrane of neurons and may be involved in regulating the transmission of glutamate, a neurotransmitter.

The gene variant linked with depression was associated with reduced SLC6A15 expression in the human hippocampus, as well as decreased volume of this brain region.

Neuron 70, 252-265 (2011)

Jürgen Schauber and Robert Besch at the Ludwig Maximilian University in Munich, Germany, and their co-workers found that, under certain conditions, DNA in the cytosol of cultured skin cells activates immune complexes called inflammasomes that contain the protein AIM2. Elevated levels of this DNA and AIM2 expression were also found in skin cells from people with psoriasis. When normal skin cells were treated with the antimicrobial peptide cathelicidin LL-37, whose production in the skin is controlled by vitamin $\mathrm{D}$, the peptide bound to cytosolic DNA, inhibiting the formation of AIM2-containing inflammasomes.

Stimulating cathelicidin production may be a promising approach for treating psoriasis, the authors suggest.

Sci. Trans. Med. 3, 82ra38 (2011)

\section{MATERIALS SCIENCE}

\section{Diamond lighter than a feather}

Aerogels are extremely porous and lightweight materials with a large surface area and many potential applications.
Peter

Pauzauskie, now at the University of Washington in Seattle, and his colleagues have created a diamond version of the material (pictured), by squeezing an aerogel of amorphous carbon until it took on a crystalline structure.

The authors used highpressure neon gas to fill and support the delicate carbon structure. They then zapped it with a laser that compressed and heated the gel, probably to more than 1,600 kelvin, until it became diamond.

Diamond aerogels could be useful as antireflective coatings, thermal conductors and other materials, the authors say.

Proc. Natl Acad. Sci. USA doi:10.1073/pnas.1010600108 (2011)

\section{$\rightarrow$ NATURE.COM}

For the latest research published by Naturevisit:

www.nature,com/latestresearch 